\title{
Estratégias para a segurança do paciente cirúrgico
}

\author{
Strategies to promote patient safety in surgical settings \\ Estrategias para la seguridad del paciente quirúrgico
}

\section{Cristina Maria Galvão ${ }^{1}$}

O processo de melhorar a segurança do paciente na prevenção de danos é complexo e desafiador. A base filosófica para o cuidado de enfermagem é fundamentada no princípio da segurança do paciente, mas com a complexidade atual da assistência à saúde, isso requer um embate contínuo para cada paciente ${ }^{(1)}$. O trabalho do enfermeiro no período perioperatório é dinâmico e exige constantemente o planejamento e a implementação de intervenções que possibilitam a prevenção de complicações decorrentes do procedimento anestésico cirúrgico visando à segurança do paciente(2).

Neste contexto entendemos que a Prática Baseada em Evidências (PBE) é uma abordagem que pode promover a segurança do paciente e envolve a definição de um problema, a busca e avaliação crítica das evidências disponíveis, implementação das evidências na prática clínica e avaliação dos resultados obtidos. A competência clínica do enfermeiro e as preferências do paciente são aspectos também incorporados nesta abordagem ${ }^{(2)}$.

A PBE é uma abordagem de solução de problema para a tomada de decisão que incorpora a busca da melhor e mais recente evidência, competência clínica do profissional e os valores e preferências do paciente dentro do contexto da saúde ${ }^{(3)}$.

A utilização de resultados de pesquisas na prática clínica é um dos pilares para a implementação da PBE na enfermagem; entretanto, consiste em processo complexo com aspectos a serem considerados, tais como: preparo do enfermeiro, ou seja, esse profissional deve desenvolver habilidades para saber como buscar, avaliar e integrar as evidências oriundas de pesquisas com os dados do paciente e as observações clínicas, acrescido a este aspecto, existe a necessidade do desenvolvimento de uma cultura organizacional, nos diferentes níveis de atenção à saúde, que favoreça a utilização de resultados de pesquisa na prática clínica.

A quantidade e complexidade de informações na área da saúde e o tempo limitado dos profissionais são aspectos que têm determinado a necessidade do desenvolvimento de métodos que proporcionem caminhos concisos até os resultados oriundos de pesquisas. A revisão sistemática e a revisão integrativa são métodos de pesquisa que permitem reunir, avaliar e sintetizar as evidências disponíveis sobre uma intervenção/tratamento ou tema/problema investigados.

Para a condução de uma revisão sistemática, o revisor elabora uma questão clínica bem delimitada. A intervenção, participantes e resultados mensurados são semelhantes nos estudos incluídos na revisão. Geralmente os estudos têm delineamento de pesquisa experimental, ou seja, são ensaios clínicos controlados randomizados (ECCR) e retratam a eficácia da intervenção investigada. Quando possível emprega-se a metanálise (os dados dos estudos são quantitativamente combinados por métodos estatísticos) ${ }^{(4)}$.

A revisão integrativa é um método de revisão mais amplo e pode ser conduzida com diferentes finalidades, por

${ }^{1}$ Enfermeira. Professora Associada da Escola de Enfermagem de Ribeirão Preto da Universidade de São Paulo, Ribeirão Preto (SP), Brasil. 
exemplo, para definição de conceitos, revisão de teorias ou síntese do estado do conhecimento de um determinado tema/problema ${ }^{(5)}$. Este método permite a inclusão de estudos conduzidos com diferentes delineamentos de pesquisa (experimental, quase-experimental e não experimental) e a identificação de lacunas que direcionam a realização de novos estudos ${ }^{()}$.

Os dois métodos de revisão podem ser utilizados como estratégias para a segurança do paciente cirúrgico, pois permitem a incorporação de evidências na prática clínica e consequentemente a melhoria da qualidade do cuidado de enfermagem. Além deste aspecto, possibilitam ao enfermeiro avaliar a qualidade das evidências disponíveis na literatura (nível de evidência) sobre a intervenção/tratamento ou tema/problema investigados, bem como fornece subsídios para a tomada de decisão no cotidiano da enfermagem perioperatória.

Para a melhor compreensão do raciocínio aqui exposto, apresentamos dois estudos desenvolvidos, um direcionado para a prevenção de hipotermia e consequentemente das complicações associadas a este evento e o outro para a prevenção de lesões no período intra-operatório. Estes estudos foram desenvolvidos utilizando como método de pesquisa a revisão sistemática e a revisão integrativa respectivamente.

A revisão sistemática teve como objetivo buscar e avaliar as evidências disponíveis sobre sistemas de aquecimento cutâneo para a prevenção de hipotermia no paciente cirúrgico durante o intra-operatório. Para tal, os critérios de inclusão delimitados foram: somente estudos com delineamento de pesquisa experimental (ensaio clínico randomizado controlado), conduzidos com pacientes submetidos a cirurgias eletivas e com idade igual ou superior a 18 anos, publicados em inglês, espanhol e português entre janeiro de 2000 e final de abril de 2007 (tecnologias novas). Os estudos que a hipotermia foi induzida ou os pacientes receberam pré-aquecimento foram excluídos. As bases de dados selecionadas foram: Medline, CINAHL, EMBASE e Cochrane Register of Controlled Trials. O número de estudos elegíveis foi 193 e frente aos critérios de inclusão e exclusão a amostra da revisão consistiu de 14 estudos. A qualidade metodológica dos ECCR foi avaliada pelo escore de Jadad e a análise dos dados foi realizada na forma descritiva. A conclusão da revisão sistemática apontou que há evidências que indicam que o cobertor elétrico de fibra de carbono tem efetividade semelhante ao sistema de ar forçado aquecido na prevenção de hipotermia; entretanto, o sistema de circulação de água aquecida é o mais efetivo na manutenção da normotermia do paciente no período intra-operatório ${ }^{(7)}$.

O objetivo da revisão integrativa foi buscar e avaliar as evidências disponíveis sobre as intervenções de enfermagem para a prevenção de lesões de pele no paciente cirúrgico no período intra-operatório, ou em decorrência desse. Os critérios de inclusão estabelecidos foram: estudos com diferentes delineamentos de pesquisa cuja metodologia adotada permitisse obter evidências fortes e moderadas (revisões sistemáticas de ECCR; ECCR individuais e estudos com delineamento quase-experimental), publicados em inglês, espanhol e português no período de 1993-2003. As bases de dados selecionadas foram Medline e CINAHL. Na amostra da revisão 14 estudos foram incluídos. Os dados evidenciados foram analisados na forma descritiva e a síntese destes permitiu apresentar ao leitor uma variedade de dispositivos utilizados para a prevenção de lesões no período intra-operatório e apontou que dentre estes, o dispositivo estático a base de polímero de viscoelástico seco é efetivo no alívio das interfaces de pressão ${ }^{(8)}$.

Acreditamos que a utilização da PBE na enfermagem proporcionaria a implantação de intervenções efetivas direcionadas para as necessidades do paciente, família e comunidade acarretando a melhoria da qualidade da assistência prestada nos diversos níveis de atenção.

A condução de métodos de revisão direcionados para os problemas vivenciados na prática clínica, bem como a utilização de revisões já publicadas consistem em estratégias que podem auxiliar na melhoria da segurança do paciente permitindo a prevenção de danos ou minimizando os riscos existentes.

\section{REFERÊNCIAS}

1. Watson DS. Patient safety. Perioper Nurs Clin. 2008;3(4):xiii.

2. Galvão CM. A prática baseada em evidências: uma contribuição para a melhoria da assistência de enfermagem perioperatória. [Tese livre-docência]. Ribeirão Preto: Escola de Enfermagem de Ribeirão Preto da Universidade de São Paulo; 2002.

3. Melnyk BM. Finding and appraising systematic reviews of clinical interventions: critical skills for evidence-based practice. Pediatr Nurs. 2003;29(2):147-9, 125.

4. Galvão CM, Sawada NO, Trevizan MA. Revisão sistemática: recurso que proporciona a incorporação das evidências na prática da enfermagem. Rev Latinoam Enferm. 2004;12(3):549-56.

5. Broome ME. Integrative literature reviews for the development of concepts. In: Rodgers BL, Knafl KA, editors. Concept development in nursing: foundations, techniques and applications. 2nd ed. Philadelphia: Saunders; c2000. p. 231-50.

6. Mendes KDS, Silveira RCCP, Galvão CM. Revisão integrativa: método de pesquisa para a incorporação de evidências na saúde e na enfermagem. Texto \& Contexto Enferm. 2008;17(4):758-64.

7. Galvão CM, Marck PB, Sawada NO, Clark AM. A systematic review of the effectiveness of cutaneous warming systems to prevent hypothermia. J Clin Nurs. 2009;18(5):627-36.

8. Ursi ES, Galvão CM. Prevenção de lesões de pele no perioperatório: revisão integrativa da literatura. Rev Latinoam Enferm. 2006;14(1):124-31. 